\title{
Excesso de peso entre adolescentes em zona rural e a alimentação escolar oferecida
}

\section{Overweight among adolescents in rural area and school meals offered}

\author{
Mariana Simões Barros ${ }^{1}$, Vania Matos Fonseca², Maria Dalva Barbosa Baker Meio², \\ Célia Regina Chaves²
}

\begin{abstract}
Resumo
Objetivo: Avaliar a composição nutricional e a aceitabilidade da alimentação escolar, o estado nutricional e a segurança alimentar dos adolescentes das escolas municipais da zona rural de Carmo no Rio de Janeiro. Métodos: Participaram 121 adolescentes de 7 escolas públicas em um município do interior do Rio de Janeiro. Foi utilizado o método de pesagem direta dos alimentos, calculando-se as porções médias oferecidas. A segurança alimentar e a aceitabilidade da alimentação escolar foram avaliadas a partir de questionários validados. O estado nutricional foi determinado pelo Índice de Massa Corporal por Idade (IMC/I) e estatura/idade e sexo. Resultados: A prevalência de sobrepeso/obesidade foi de 28,9\%, baixo peso 1,7\% e baixa estatura por idade $0,8 \%$. Todas as escolas ofereceram valores de nutrientes superiores à recomendação, exceto para fibras, vitamina C, magnésio e cálcio. A oferta de alimentos de elevada densidade calórica foi alta. A maioria dos alunos $(92 \%)$ com insegurança alimentar consumia a alimentação escolar e nenhum destes possuía baixo peso ou estatura. Conclusão: Os alunos apresentaram um bom estado nutricional, mas sendo as prevalências de sobrepeso/obesidade próximas àquelas de áreas urbanas. É necessária maior ênfase na educação nutricional e planejamento da alimentação escolar sobre os grupos de alimentos oferecidos.
\end{abstract}

Palavras-chave: alimentação escolar; segurança alimentar e nutricional; adolescente.

\begin{abstract}
Objective: To evaluate the nutricional composition and the acceptability of the school meals, nutritional status, and food security of adolescents in rural public schools in Carmo, Rio de Janeiro. Methods: One hundred and twenty-one adolescents from the 7 public schools of a country side city of Rio de Janeiro, participated in the study. The method of food direct weighing was utilized, where the offered average portions were calculated. Food security and school meals acceptability were evaluate by validated questionnaires. The nutricional status was determined by Body mass index (BMI for age) and height for age and gender. Results: The occurrence of overweight/obesity was $28.9 \%$, underweight $1.7 \%$ and low height-for-age $0.8 \%$. All school menus offered higher nutritional values than the recommended, except for fiber, vitamin $\mathrm{C}$, magnesium, and calcium. The offered of high energetic density food was high. The majority of the students (92\%) with food insecurity ate the meals offered by the school and none of them were low weight-for-age or low height-for-age. Conclusion: The students presented a good nutritional status, but the prevalence of overweight/obesity was similar to those in urban areas. It is required more emphasis on nutrition education, and planning of the school meals of the group of food to be selected.
\end{abstract}

Keywords: school feeding; food security; adolescent.

Trabalho realizado no Instituto Fernandes Figueira (IFF) da Fundação Oswaldo Cruz (FIOCRUZ) - Rio de Janeiro (RJ), Brasil.

${ }^{1}$ Mestre pelo IFF da FIOCRUZ - Rio de Janeiro (RJ), Brasil.

${ }^{2}$ Doutora pelo IFF da FIOCRUZ - Rio de Janeiro (RJ), Brasil.

Endereço para correspondência: Mariana Simões Barros - Direção Instituto Fernandes Figueira - Avenida Rui Barbosa, 716 - CEP: $22250-020$ -

Rio de Janeiro (RJ), Brasil - E-mail: marysb@iff.fiocruz.br

Fonte de financiamento: nenhuma.

Conflito de interesse: nada a declarar. 


\section{INTRODUÇÃO}

O objetivo principal da alimentação escolar é suprir parcialmente as necessidades nutricionais diárias dos alunos, $\mathrm{e}$, além disso, contribuir para a formação de bons hábitos alimentares, evitando a evasão escolar, e corrigindo deficiências alimentares comuns, que repercutem na saúde física e mental da criança e no processo de aprendizagem, pois crianças e adolescentes desnutridos apresentam baixo rendimento escolar ${ }^{1}$. A qualidade da alimentação escolar ganha relevância social ainda maior considerando o número de alunos que têm nesta alimentação a única opção de refeição diária².

A nova lei que estabelece o programa de alimentação escolar diz:

que o Ministério da Educação pode propor ações educativas que perpassem pelo currículo escolar, abordando o tema alimentação e nutrição e o desenvolvimento de práticas saudáveis de vida, na perspectiva da segurança alimentar e nutricional ${ }^{3}$.

Segurança alimentar e nutricional (SAN) é a garantia do direito ao acesso regular e permanente a alimentos de qualidade, em quantidade suficiente, sem comprometer o acesso a outras necessidades essenciais, tendo como base, práticas alimentares promotoras de saúde que respeitem a diversidade cultural e que sejam ambiental, cultural, econômica e socialmente sustentáveis ${ }^{4}$. A insegurança alimentar pode resultar em baixa qualidade da dieta, interferindo no estado nutricional do adolescente ${ }^{5,6}$.

Em estudos realizados no Sudeste e no Nordeste do Brasil, a prevalência da desnutrição na área rural foi superior a da área urbana $^{7}$, sendo encontrado o oposto quanto ao sobrepeso e a obesidade $^{8}$. Segundo o Instituto Brasileiro de Geografia e Estatística $(\mathrm{IBGE})^{9}$, a prevalência de sobrepeso/obesidade em adolescentes tendeu a ser mais alta no meio urbano do que no rural.

De acordo com a Pesquisa Nacional de Demografia e Saúde da Criança e da Mulher ${ }^{10}$, nas áreas rurais do Sudeste, $98,2 \%$ dos adolescentes entre 11 e 14 anos frequentam a escola. Mesmo assim existem poucos estudos sobre a alimentação escolar em zonas rurais e sua influência para o estado nutricional dos estudantes.

O objetivo deste trabalho foi determinar a composição nutricional e aceitabilidade da alimentação escolar oferecida, o estado nutricional dos adolescentes das escolas municipais da zona rural de um município do interior do Rio de Janeiro e a segurança alimentar de suas famílias. E ainda verificar as associações entre aceitabilidade da alimentação escolar, insegurança alimentar e o estado nutricional dos adolescentes. Trata-se de uma oportunidade de conhecer melhor a realidade da alimentação de escolas rurais, e ainda contribuir na discussão das mudanças necessárias para o controle do aumento do excesso de peso dos escolares, no momento da ocorrência de reformulação destas atividades por parte do poder público municipal.

\section{MÉTODOS}

Foi realizado um estudo observacional do tipo transversal com 121 adolescentes de ambos os sexos em todas as 7 unidades escolares municipais de ensino fundamental da zona rural no município do Carmo, pertencente à Região Serrana do estado do Rio de Janeiro, no período do $2^{\circ}$ semestre de 2008. De acordo com o IBGE ${ }^{11}$, em 2007, Carmo tinha uma população de 16.690 habitantes e uma densidade demográfica de 48 habitantes por $\mathrm{km}^{2}$. A rede municipal possuía 17 escolas, sendo 10 na área urbana e 7 na área rural.

Foram incluídas no estudo todas as escolas municipais que possuíam alunos matriculados no $5^{\circ}$ ano do $1^{\circ}$ segmento do ensino fundamental e do $6^{\circ}$ ao $9^{\circ}$ anos do $2^{\circ}$ segmento do ensino fundamental da zona rural, perfazendo um total de 7 escolas, com 144 estudantes na faixa de idade entre 10 e 19 anos, de ambos os sexos. Destes, apenas 121 alunos foram avaliados, pois 6 estiveram ausentes no dia de entrega dos termos de consentimento livre e esclarecido (TCLE) e questionários para os responsáveis, 4 estiveram ausentes no momento de coleta de dados antropométricos, e, em 14 deles, os pais se recusaram a assinar o termo de consentimento. Nenhuma adolescente estava grávida no momento do estudo.

Foram realizadas duas visitas em dias não consecutivos e aleatórios em cada escola e em todos os turnos. Durante cada visita foram preenchidas fichas técnicas de composição nutricional e peso dos ingredientes utilizados em cada preparação oferecida nos cardápios dos dias visitados. Foi utilizado o método de pesagem direta de alimentos no qual foram pesadas separadamente as porções médias de cada preparação oferecida aos alunos. Os alimentos sólidos foram pesados em uma balança digital da marca Plenna, com capacidade de aferição de $5 \mathrm{~kg}$ e graduação de $1 \mathrm{~g}$, enquanto os líquidos foram medidos em recipiente com graduação de $10 \mathrm{~mL}$ e capacidade máxima de $500 \mathrm{~mL}$.

As recomendações nutricionais contidas na Resolução/ CD/FNDE $n^{\circ} 38$, de 16 de julho de $2009^{3}$, foram utilizadas para a comparação entre os nutrientes da merenda oferecida e as necessidades nutricionais diárias recomendadas para cada estrato etário.

A qualidade de respostas às entrevistas e a capacidade de respostas a questionários melhoram com a idade, sendo que a partir dos sete anos de idade há um aumento na confiabilidade destas respostas ${ }^{12}$. Por isso optou-se em utilizar o questionário de aceitabilidade ${ }^{13} \mathrm{com}$ os alunos, contendo questões 
sobre preferências alimentares e alimentação saudável, considerando que estes já estejam aptos a responder o questionário de forma confiável.

$\mathrm{Na}$ avaliação do grau de insegurança alimentar das famílias dos escolares, foi enviado para preenchimento pelos pais ou responsáveis o questionário de avaliação da segurança e insegurança alimentar das famílias brasileiras - Escala Brasileira de Insegurança Alimentar (EBIA) ${ }^{14}$, sendo criadas as categorias segurança e insegurança alimentar.

$\mathrm{O}$ índice de massa corporal (peso $(\mathrm{kg}) /$ estatura X estatura $\left(\mathrm{m}^{2}\right)$ ), segundo sexo e idade (IMC/I), e o índice de estatura para idade (E/I) foram utilizados para avaliação do estado nutricional. O peso corporal e a estatura foram aferidos com o aluno descalço, usando roupas leves, com os braços relaxados ao longo do corpo ${ }^{15}$, em balança portátil com antropômetro acoplado da marca Líder. Todas as mensurações foram feitas em duplicata pela própria pesquisadora.

Calculou-se o IMC/I e o índice E/I utilizando-se o software AnthroPlus for Personal Computers ${ }^{16}$. Os pontos de corte para classificação do estado nutricional utilizados foram: maior que +2 escore $Z$ (obesidade), entre +1 e +2 escore $Z$ (sobrepeso), entre $-2 \mathrm{e}+1$ escore $Z$ (eutrofia) e menor que -2 escore $Z$ (baixo peso para sexo e idade); e para o índice E/I foi considerado déficit de estatura para sexo e idade -2 escore $Z^{17}$. Em alguns casos foram somados sobrepeso e obesidade na apresentação do resultado.

O grau de maturação sexual foi aferido durante as mensurações antropométricas, quando as meninas relatavam a idade da menarca, e nos meninos era verificada, sempre pelo mesmo avaliador, a presença de pelos axilares ${ }^{18}$. A menarca pode ser relacionada à fase final da maturação sexual das meninas ${ }^{19}$. No caso dos meninos, o surgimento dos pelos axilares ocorre logo após o pico de velocidade em estatura e precede o estágio final de maturação sexual ${ }^{20}$. Concluindo assim que esta classificação ficou finalmente categorizada em púbere (com menarca ou pelos axilares) e pré-púbere (antes destes sinais).

Para construção do banco de dados e análises estatísticas foi utilizado o programa Epi Info $2000^{21}$, onde foram comparados dados obtidos em cada escola, e analisadas as prevalências dos indicadores selecionados nos objetivos. Nas comparações feitas através das prevalências e médias das variáveis estudadas na população, não foram realizados testes para avaliar a significância estatística por se tratar de um censo abrangendo todos os escolares. As informações sobre a composição nutricional da merenda escolar foram analisadas utilizando o software Avanutri versão online ${ }^{22}$, do qual foram selecionadas a Tabela de composição de alimentos ${ }^{23} \mathrm{e}$ a Tabela Brasileira de Composição de Alimentos ${ }^{24}$.
Esta pesquisa foi aprovada pelo Comitê de Ética em Pesquisa do Instituto Fernandes Figueira/Fundação Oswaldo Cruz (protocolo $\mathrm{n}^{\circ}$ 0035.0.008.000-08), e tanto os responsáveis, quanto os adolescentes incluídos no estudo assinaram o termo de consentimento informado.

\section{RESULTADOS}

Dos 121 alunos estudados, 69 eram do sexo feminino (57\%) e 52 do sexo masculino (43\%). A média de idade foi de 12 anos. Quanto ao estado nutricional, somente 2 (1,7\%) apresentaram baixo peso, sendo um de cada sexo, e somente 1 aluno $(0,8 \%)$ apresentou déficit de estatura para idade. A prevalência de sobrepeso/obesidade foi de $28,9 \%$, sendo $30,8 \%$ dos meninos e 27,6\% das meninas (Tabela 1).

Entre os alunos avaliados, $62 \%$ estavam em estágios iniciais de maturação sexual (54\% dos meninos e $66 \%$ das meninas). Dos 75 em estágios iniciais de maturação sexual, $26,7 \%$ tinham sobrepeso/obesidade, enquanto entre os 45 alunos que tinham maturidade sexual, este percentual foi de 33,3\%. Das meninas em estágios iniciais de maturação sexual, 20,0\% tinham sobrepeso/obesidade, enquanto $43,5 \%$ das que tinham maturidade sexual se encontravam em tais classificações. Quanto aos meninos, os em estágios iniciais de maturação sexual com sobrepeso/obesidade somam 36,7\%, enquanto os que tinham maturidade sexual perfazem $23,8 \%$.

Todas as escolas ofereceram, nos seus cardápios, valores de nutrientes superiores à recomendação de 15\% das necessidades diárias dos alunos das faixas etárias estudadas (Tabela 2). Verifica-se variação de nutrientes entre escolas, sendo que a quantidade calórica oferecida variou entre as escolas de 574,30 a $1.195 \mathrm{kcal}$, mas não houve grande variação entre os nutrientes na comparação dos 2 dias avaliados em cada escola. A média de calorias provenientes de lipídeos correspondeu a $29,3 \%$ do total, sendo $9,7 \%$ de gordura saturada. Foram adicionados em média dois gramas de sal/per capita.

Em todas as escolas foi oferecido diariamente pão com margarina e leite com achocolatado (Figura 1). O feijão foi o $3^{\circ}$ alimento mais consumido (78\%), seguido da carne bovina

Tabela 1. Estado nutricional segundo sexo dos alunos adolescentes das escolas municipais da zona rural de Carmo, Rio de Janeiro, 2008

\begin{tabular}{lcccc}
$\begin{array}{l}\text { Estado nutricional } \\
\text { segundo IMC/I }\end{array}$ & $\begin{array}{c}\text { Masculino } \\
\mathbf{n}(\%)\end{array}$ & IC95\% & $\begin{array}{c}\text { Feminino } \\
\mathbf{n}(\%)\end{array}$ & IC95\% \\
Baixo peso & $1(1,9)$ & $0-10,3$ & $1(1,4)$ & $0-7,8$ \\
Eutrofia & $35(67,3)$ & $52,9-79,7$ & $49(71,0)$ & $58,8-81,3$ \\
Sobrepeso & $7(13,5)$ & $5,6-25,8$ & $13(18,9)$ & $10,4-30,1$ \\
Obesidade & $9(17,3)$ & $8,2-30,3$ & $6(8,7)$ & $3,3-18,0$ \\
\hline
\end{tabular}

IMC/I: índice de massa corporal por idade; IC95\%: intervalo de confiança de 95\% 
(57\%) e do arroz (50\%). Das frutas, só estiveram presentes nos 14 cardápios analisados, banana $(7,1 \%)$ e maçã $(21,4 \%)$. Somente $35,7 \%$ dos cardápios ofereceram legumes e verduras nas suas preparações, e apenas 3 cardápios (21\%) continham salada crua.

Do total de alunos, 33 (27,3\%) relataram também levar alimentos de casa, sendo que destes, 69,7\% levavam biscoitos, $36,4 \%$ salgados fritos, $24,3 \%$ refrigerante e somente $15 \%$ frutas.

Entre os 35 alunos com sobrepeso/obesidade, 36\% não consumiam a alimentação escolar; entre os que consumiam esta prevalência era de $26 \%$.

Entre os jovens que não consumiam verduras e legumes pelo menos 5 vezes por semana, a prevalência de sobrepeso/obesidade variou segundo o sexo: $31,8 \%$ (masculino) e $25,7 \%$ (feminino). Já para aqueles que consumiam, a prevalência foi de $25,8 \%$ e $28,3 \%$ respectivamente.

Insegurança alimentar foi detectada em $35,6 \%$ das famílias dos alunos, sendo 25,7\% insegurança alimentar leve, 6,9\% insegurança alimentar moderada e 3,0\% insegurança alimentar grave.

Dentre as meninas, $42,1 \%$ cujas famílias apresentaram algum grau de insegurança alimentar apresentaram sobrepeso/obesidade; entre aquelas com segurança alimentar, a prevalência foi de 30,6\%. Quanto aos meninos, 26,7\% com algum grau de insegurança alimentar apresentaram sobrepeso/obesidade; a prevalência foi de $35,7 \%$ entre os que viviam em segurança alimentar (Tabela 3 ).

Das 69 famílias de meninas, 30,6\% relataram ter ficado sem dinheiro para ter uma alimentação saudável e variada nos últimos 3 meses, sendo que entre as famílias de meninos esta prevalência foi de $18,9 \%$.
Dentre os pais de alunos com sobrepeso/obesidade, $27,1 \%$ relataram que nos últimos 3 meses ficaram sem dinheiro para ter uma alimentação saudável e variada; $25 \%$ das famílias dos eutróficos passaram por tal situação.

A prevalência de adesão à alimentação escolar foi de $88,4 \%$ (92/104) e sua aceitação foi de 85,5\% (89/104) (Tabela 4). A maioria dos alunos (92\%) que apresentaram algum nível de insegurança alimentar consumia a alimentação escolar, exceto 3 alunos com insegurança alimentar que relataram não comerem nenhum dia por não gostarem da comida oferecida.

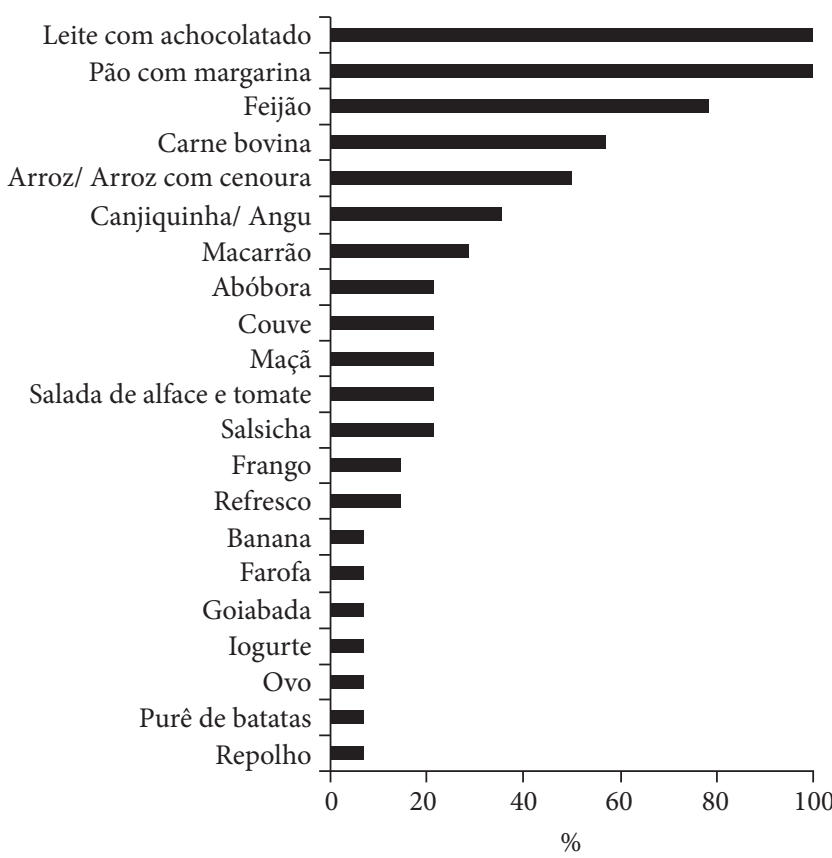

Figura 1. Frequência de consumo dos alimentos utilizados na merenda escolar em Carmo, Rio de Janeiro, 2008

Tabela 2. Composição média dos nutrientes da alimentação escolar a partir de 2 cardápios de cada escola oferecidos pelas escolas municipais da zona rural de Carmo, Rio de Janeiro, 2008

\begin{tabular}{|c|c|c|c|c|c|c|c|c|c|c|c|}
\hline 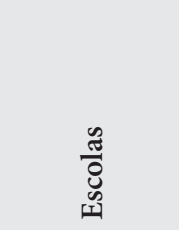 & ت્ఝี & 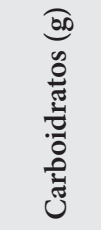 & $\begin{array}{l}\text { क्रि } \\
\Xi \\
\Xi\end{array}$ & 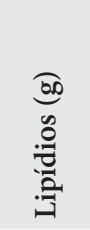 & 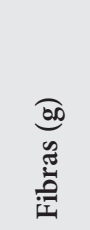 & 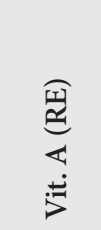 & 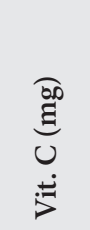 & $\underset{\tilde{J}}{\stackrel{000}{\Xi}}$ & 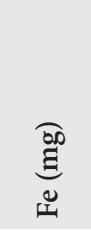 & $\underbrace{\underset{\Xi 00}{\Xi}}_{\sum_{i}^{\infty}}$ & 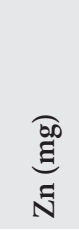 \\
\hline 1 & 574,30 & 96,4 & 23,79 & 10,40 & 7,825 & 76,87 & 1,75 & 241,10 & 3,96 & 67,73 & 3,34 \\
\hline 2 & 723,80 & 112,21 & 27,88 & 18,16 & 8,215 & 402,00 & 10,91 & 423,40 & 4,99 & 61,59 & 2,83 \\
\hline 3 & 499,65 & 63,73 & 18,80 & 18,87 & 4,75 & 233,60 & 8,27 & 228,90 & 2,17 & 45,95 & 0,94 \\
\hline 4 & 1195,00 & 154,87 & 46,96 & 43,08 & 10,7 & 149,10 & 24,51 & 630,40 & 5,35 & 80,05 & 5,64 \\
\hline 5 & 687,70 & 91,65 & 28,64 & 22,97 & 4,46 & 168,90 & 21,95 & 309,70 & 3,49 & 52,94 & 5,51 \\
\hline 6 & 737,40 & 89,73 & 27,58 & 29,81 & 5,73 & 162,50 & 19,51 & 417,20 & 4,07 & 74,94 & 3,78 \\
\hline 7 & 996,20 & 141,53 & 32,85 & 33,21 & 7,895 & 644,00 & 7,83 & 391,90 & 5,14 & 59,43 & 3,52 \\
\hline Média global & 773,44 & 107,16 & 29,50 & 25,21 & 7,08 & 262,42 & 13,53 & 377,51 & 4,16 & 63,23 & 3,65 \\
\hline$\%$ Vet & 35,54 & 30,31 & 43,38 & 46,25 & 23,21 & 37,49 & 22,55 & 29,04 & 39,62 & 20,07 & 40,55 \\
\hline
\end{tabular}

Vet: porcentagem do valor energético total; Ptn: proteína; Vit. A (RE): vitamina A (retinol); Vit. C: vitamina C 
Nenhum aluno pesquisado com algum grau de insegurança alimentar apresentou baixo peso.

\section{DISCUSSÃO}

A escola, por ser um espaço que crianças frequentam, é um lugar privilegiado para a obtenção de informações que possibilitem a identificação da distribuição dos distúrbios nutricionais, o monitoramento das desigualdades sociais em saúde e a identificação de necessidades de implementação de ações específicas de nutrição e saúde ${ }^{25}$.

A prevalência de sobrepeso/obesidade nas escolas rurais estudadas foi de $28,9 \%$, com taxas semelhantes entre meninos $(30,8 \%)$ e meninas $(27,6 \%)$. Estes resultados são muito superiores ao encontrado em adolescentes entre 15 e 19 anos

Tabela 3. Insegurança alimentar e estado nutricional segundo sexo dos alunos adolescentes das escolas municipais da zona rural de Carmo, Rio de Janeiro, 2008

\begin{tabular}{lcccc} 
& \multicolumn{2}{c}{$\begin{array}{c}\text { Insegurança } \\
\text { Alimentar }\end{array}$} & \multicolumn{2}{c}{$\begin{array}{c}\text { Segurança } \\
\text { Alimentar }\end{array}$} \\
\cline { 2 - 5 } Estado nutricional & $\mathbf{n}(\%)$ & IC95\% & n (\%) & IC95\% \\
Masculino & & & & \\
$\quad$ Sobrepeso/obesidade & $4(26,7)$ & $7,8-55,1$ & $10(35,7)$ & $17,9-54,3$ \\
$\quad$ Eutrófico & $11(73,3)$ & $44,9-92,2$ & $18(64,3)$ & $42,3-79,3$ \\
Feminino & & & & \\
$\quad$ Sobrepeso/obesidade & $8(42,1)$ & $20,3-66,5$ & $11(30,6)$ & $15,9-47,0$ \\
Eutrófico & $11(57,9)$ & $33,5-79,7$ & $25(69,4)$ & $50,2-82,0$ \\
\hline
\end{tabular}

IC95\%: intervalo de confiança de 95\%

Tabela 4. Prevalência de insegurança alimentar das famílias e aceitação da alimentação escolar dos alunos adolescentes das escolas municipais de Carmo, Rio de Janeiro, 2008

\begin{tabular}{lcccc}
\multirow{2}{*}{$\begin{array}{l}\text { Aceitação da } \\
\text { alimentação escolar }\end{array}$} & \multicolumn{2}{c}{$\begin{array}{c}\text { Insegurança } \\
\text { alimentar }\end{array}$} & \multicolumn{2}{c}{$\begin{array}{c}\text { Segurança } \\
\text { alimentar }\end{array}$} \\
\cline { 2 - 5 } & $\mathbf{n}(\%)$ & IC95\% & n (\%) & IC95\% \\
$\begin{array}{l}\text { Come a merenda } \\
\text { oferecida na escola }\end{array}$ & & & & \\
$\quad$ Não & $3(25,0)$ & $5,5-57,2$ & $9(75,0)$ & $42,8-94,5$ \\
Sim & $34(37,0)$ & $27,1-47,7$ & $58(63,0)$ & $52,3-72,9$ \\
Total & $37(35,6)$ & & $67(64,4)$ &
\end{tabular}

Gosta da comida

oferecida na escola

$\begin{array}{lcccc}\text { Não } & 3(20,0) & 4,3-48,1 & 12(80,0) & 51,9-95,7 \\ \text { Sim } & 34(38,2) & 28,1-49,1 & 55(61,8) & 50,9-71,9 \\ \text { Total } & 37(35,6) & & 67(64,4) & \end{array}$

Dias na semana que come a merenda da escola

\begin{tabular}{lcccc} 
Nenhum & $3(21,4)$ & $4,7-50,8$ & $11(78,6)$ & $49,2-95,3$ \\
Alguns dias & $17(31,5)$ & $19,5-45,6$ & $37(68,5)$ & $54,4-80,5$ \\
Total & $17(47,2)$ & $35,5-69,6$ & $19(52,8)$ & $35,5-69,6$ \\
\hline
\end{tabular}

IC95\%: intervalo de confiança de 95\% no Sudeste rural do Brasil 9,2\% ${ }^{8}$. Em estudo realizado em escolares com idade entre 10 e 17 anos nas áreas urbanas do Rio de Janeiro, foram encontradas prevalências de sobrepeso/obesidade também menores, sendo $22,4 \%$ para meninos e $24,5 \%$ para meninas ${ }^{26}$.

Segundo o IBGE$^{9}$, a prevalência de sobrepeso/obesidade em meninas é de 23,9\% no Sudeste rural e de 25,7\% no Sudeste urbano. Nossos resultados mostraram taxas para meninas maiores do que as relatadas tanto para a área rural quanto para as áreas urbanas do Sudeste.

Em estudos com escolares de dois municípios no interior do Rio Grande do Sul, o excesso de peso foi mais frequente na zona rural que na zona urbana ${ }^{27,28}$, mesmo assim foram menores que o encontrado no presente estudo.

A prevalência de sobrepeso/obesidade encontrada foi maior entre as meninas que tinham maturidade sexual, em relação às meninas em estágios iniciais de maturação sexual, também observado por Oliveira e Veiga ${ }^{29}$, o que seria esperado, pois a maior parte do crescimento em estatura ocorre antes da maturação sexual, sendo o ganho de peso mais acelerado após a maturação. Tal fato não ocorreu entre os meninos, onde a prevalência de sobrepeso/obesidade foi maior entre os que estavam em estágios de maturação sexuais iniciais $(36,7 \%)$ em relação aos em estágios finais (23,8\%). O marcador usado neste estudo para avaliação do grau de maturação sexual, presença de pelos axilares, os quais surgem logo após o pico de velocidade em estatura, e precede o estágio final de maturação sexual masculina ${ }^{20}$, pode indicar que alguns dos adolescentes pré-púberes que apresentaram excesso de peso, podem já ser púberes, porém não estarem ainda na fase deste pico da velocidade de crescimento, e, portanto não apresentarem ainda pelos axilares.

O estudo de Linhares et al. ${ }^{30}$, com 136 meninos em Juiz de Fora, Minas Gerais (MG), na faixa etária de 10 a 14 anos, mostrou que, embora houvesse aumento no peso e na estatura com a progressão da maturação sexual, a diminuição do IMC entre o estágio 2 e o estágio 3 de maturação sexual (avaliada pela distribuição dos pelos pubianos e pelo estágio de desenvolvimento da genitália externa), poderia estar relacionada ao aumento proporcionalmente maior da estatura sobre o peso, nessa fase de crescimento muito acelerado, uma vez que, no estágio 4, os indivíduos voltam ao padrão anterior de aumento dos parâmetros antropométricos.

O déficit de crescimento (índice de estatura/idade) foi encontrado somente nos meninos, com uma prevalência de $0,8 \%$, muito abaixo das prevalências para adolescentes brasileiros $(3,4 \%)$, inclusive do Sudeste rural (2,3\% para meninos e $2,0 \%$ para meninas $)^{9}$. Este índice, embora sofra influência do ritmo de maturação sexual, reflete em boa medida o histórico 
do desempenho do crescimento linear dos indivíduos ${ }^{31}$, refletindo, portanto, um bom estado nutricional destes estudantes em longo prazo. A prevalência da desnutrição na área rural brasileira é apontada como superior à da área urbana, constatada na pesquisa populacional sobre padrões de vida realizada em $1997^{7}$, a qual indicou um déficit de estatura de $8,4 \% \mathrm{em}$ crianças de 7 a 10 anos no Sudeste rural e 12,0\% no Sudeste urbano. Entretanto, nosso resultado mostra 2,5 vezes menos déficit de estatura/idade quando comparado com o Sudeste rural, mostrando que o déficit nutricional crônico não é um problema entre as crianças estudadas, e sim a ocorrência de sobrepeso/obesidade.

Todas as escolas avaliadas oferecem desjejum ou lanche e almoço aos estudantes, sendo sempre duas refeições. Em todas as escolas, os valores encontrados dos nutrientes avaliados na merenda oferecida foram superiores aos $15 \%$ das necessidades diárias dos estudantes, que era preconizado pela legislação brasileira na época da coleta dos dados ${ }^{4}$, sendo que de acordo com a recente Resolução de 16 de julho de $2009^{3}$, as escolas devem passar a suprir no mínimo, 30\% (trinta por cento) das necessidades nutricionais diárias aos alunos matriculados na educação básica, em período parcial quando ofertadas duas ou mais refeições. Nos nossos resultados, as escolas rurais ofereceram 35,5\% das necessidades diárias de calorias, e mais de 30\% para alguns nutrientes, exceto para as fibras, vitamina $\mathrm{C}$, magnésio e cálcio, onde a oferta estava abaixo dos 30\% agora preconizados. Encontramos ainda $29,3 \%$ calorias provenientes de lipídios, sendo 9,7\% de gordura saturada, sendo que os valores recomendados pela legislação ${ }^{3}$ são de $15-30 \%$ para gorduras totais e no máximo $10 \%$ para gordura saturada. Portanto, as escolas do município do Carmo estavam oferecendo uma alimentação de acordo com as recomendações calóricas provenientes das gorduras.

Entretanto, ao analisarmos os alimentos oferecidos nas merendas, constatamos inadequação na distribuição dos tipos de alimentos. A maior frequência de oferta de alimentos de elevada densidade calórica na merenda, como goiabada, achocolatado, arroz, canjiquinha, e a oferta muito baixa de frutas, legumes e verduras nestas escolas, podem estar associadas às maiores prevalências de sobrepeso/obesidade encontradas nestes estudantes de área rural. Tal fato é bastante preocupante, pois, um dos objetivos da merenda escolar é a formação dos hábitos alimentares saudáveis ${ }^{32}$, e o consumo de alimentos de alta densidade energética entre adolescentes propicia o desenvolvimento de excesso de peso e podem ser determinantes de obesidade e doenças crônicas não transmissíveis na fase adulta $^{33}$.

Assim como no presente estudo, uma pesquisa realizada em Juiz de Fora, $\mathrm{MG}^{34}$, apresentou como principais componentes dos cardápios das escolas públicas o arroz, macarrão, canjiquinha, angu, pão com margarina e achocolatado. Segundo o IBGE ${ }^{35}$, a participação de frutas, verduras e legumes na dieta da população do meio urbano $(2,6 \%)$ foi duas vezes maior que a das áreas rurais $(1,3 \%)$, embora, esta taxa de consumo nas cidades esteja bastante aquém das recomendações de 6-7\% para este grupo de alimentos nas calorias totais consumidas; e que seria esperado exatamente o oposto, um maior consumo destes alimentos em populações rurais. Foram adicionados em média, na principal refeição oferecida nas escolas (almoço), cerca de dois gramas de sal/per capita, sendo esta quantidade o dobro da recomendação diária segundo a Resolução/CD/FNDE n 38, de 16 de julho de $2009^{3}$.

Outro aspecto importante é que a prevalência de sobrepeso encontrada foi maior entre os alunos que não consomem a merenda escolar, possivelmente porque entre estes estudantes foi declarado um consumo regular de alimentos na merenda levada de casa do tipo: biscoitos (69,7\%), frituras (36,4\%), e refrigerantes $(24,2 \%)$.

Nossos resultados mostraram que a adesão (consumo da merenda, $89,2 \%$ ) e aceitação (gostar da merenda, 85,6\%) foram altas. Apenas $34,6 \%$ dos alunos relataram comer a merenda todos os dias, pois a maioria não gosta de algumas preparações como: canjiquinha (20,7\%), sopa (16,5\%) e macarrão $(14,0 \%)$. Outro estudo ${ }^{36}$ mostrou que dentre as crianças pertencentes ao Programa Bolsa Família a adesão diária à merenda foi $71,4 \%$, apontando que a merenda nem sempre era do gosto destes.

Das famílias avaliadas, 35,6\% possuía algum grau de insegurança alimentar, valor próximo do encontrado no Sudeste rural (32,9\%) de acordo com a Pesquisa Nacional de Demografia e Saúde da Criança e da Mulher ${ }^{10}$. A maioria dos alunos (92\%) com insegurança consumia a merenda oferecida pela escola.

A proporção de adolescentes com sobrepeso/obesidade entre os que apresentavam algum grau de insegurança alimentar foi alta $(35,3 \%)$, sendo mais elevada que a prevalência encontrada por Lopes ${ }^{37}$, em outro estudo, em Duque de Caxias, Rio de Janeiro, com 523 adolescentes de 12 a 18 anos $(20,1 \%)$. Poucos estudos nacionais analisaram a relação entre estes dois fatores, insegurança alimentar e sua associação com sobrepeso/obesidade. A insegurança alimentar prejudica a qualidade da alimentação, e pode estar relacionada ao consumo mais frequente de alimentos de baixo custo e alta densidade energética, ou mesmo à frequente restrição alimentar, o que pode levar ao consumo excessivo nos períodos de acesso regular $^{38,39}$. A baixa qualidade da dieta poderia explicar, neste estudo, a prevalência de sobrepeso/obesidade maior entre as meninas adolescentes que apresentavam algum grau de 
insegurança alimentar $(42,1 \%)$ em relação as que apresentavam segurança alimentar (30,6\%). Tal achado foi relatado em outro estudo ${ }^{6}$ com adolescentes norte-americanos, com 6.995 crianças e adolescentes entre 3 e 17 anos, que encontrou maior risco de sobrepeso e sobrepeso em famílias com insegurança alimentar. Alaimo et al. ${ }^{40}$, ao investigar associação entre insuficiência alimentar e excesso de peso entre 5.200 crianças com 2 a 7 anos e 3.996 adolescentes americanos com 8 a 16 anos, verificou que as adolescentes brancas não hispânicas em situação de insuficiência alimentar apresentaram 3,5 vezes mais riscos de excesso de peso do que as em suficiência alimentar. Outro estudo de Casey et al. ${ }^{5}$, com 5.669 crianças e adolescentes americanos, mostrou que famílias de baixa renda em situação de insuficiência alimentar apresentavam menor consumo de calorias, carboidratos e frutas, ingestão de alto índice de colesterol e maior prevalência de excesso de peso, quando comparadas às famílias com suficiência alimentar.

A Secretaria Municipal de Educação do município tinha recém-contratado uma nutricionista para o planejamento e acompanhamento da merenda escolar, mas no momento da coleta de dados, a mesma não havia realizado nenhuma alteração. Os resultados deste trabalho são de interesse destes gestores e serão apresentados às equipes das escolas e seus dirigentes, visando ações de mudanças que possibilitem intervenções.
Uma limitação do presente estudo diz respeito ao marcador que foi utilizado para avaliação da maturação sexual nos meninos, pelos axilares, em detrimento do uso das pranchas de Tanner, recomendadas pela Organização Mundial da Saúde (OMS) ${ }^{15}$ como instrumento na avaliação para este fim; tal fato ocorreu por estas não terem sido autorizadas para aplicação nas escolas.

\section{CONCLUSÃO}

Os alunos destas escolas rurais apresentaram prevalência de sobrepeso/obesidade, próximas àquelas de áreas urbanas, fator preocupante a ser destacado. $\mathrm{O}$ maior consumo de alimentos de alta densidade na merenda consumida pode estar associado a estes achados, mostrando a necessidade de substituição dos mesmos por frutas, legumes e verduras, os quais apresentaram baixa frequência de consumo, apesar de estas escolas estarem situadas em uma área rural. Nenhum dos alunos avaliados, com algum grau de insegurança alimentar, apresentou baixo peso, ao contrário, alguns tinham sobrepeso/obesidade, possivelmente relacionada à qualidade de suas dietas. $\mathrm{O}$ que aumenta a importância destas escolas darem uma maior ênfase na educação nutricional entre os estudantes, investindo na formação de hábitos alimentares mais saudáveis, e ainda orientarem os responsáveis pelo planejamento da merenda sobre os grupos de alimentos oferecidos, em função deste aumento das taxas de sobrepeso.

\section{REFERÊNCIAS}

1. Brasil. Ministério da Educação. Resolução/CD/FNDE nº 32 de 10 de agosto de 2006: Estabelecer as normas para a execução do Programa Nacional de Alimentação Escolar - PNAE. Diário Oficial da União 2006; 10 ago.

2. Prass FS, Moreira TR, Oliveira VR. Contribuição do Programa Nacional de Alimentação Escolar sobre a condição nutricional de crianças em idade escolar. Hig Alimen. 2007;21(149):22-6.

3. Brasil. Ministério da Educação. Resolução/CD/FNDE no 38 de 16 de julho de 2009: Dispõe sobre o atendimento da alimentação escolar aos alunos da educação básica no Programa Nacional de Alimentação Escolar - PNAE. Diário Oficial da União 2009; 16 jul.

4. Brasil. Presidência da República. Lei no 11.346 de 15 de setembro de 2006: Cria o Sistema Nacional de Segurança Alimentar e Nutricional (SISAN) com vistas em assegurar o direito humano à alimentação adequada e dá outras providências. Casa Civil 2006; 15 set.

5. Casey PH, Szeto K, Lensing S, Bogle M, Judy Weber J. Children in foodinsufficient, low-income families: prevalence, health, and nutrition status. Arch Pediatr Adolesc Med. 2001;155(4):508-14.

6. Casey PH, Simpson PM, Gossett JM, Bogle ML, Champagne CM, Connell C, et al. The Association of Child and Household Food Insecurity With Childhood Overweight Status. Pediatrics. 2006;118(5):1406-13.

7. Burlandy L, Anjos LA. Acesso à alimentação escolar e estado nutricional de escolares no Nordeste e Sudeste do Brasil, 1997. Cad Saúde Pública. 2007;23(5):1217-26.
8. Magalhães VC, Azevedo G, Mendonça S. Prevalência e fatores associados a sobrepeso e obesidade em adolescentes de 15 a 19 anos das regiões Nordeste e Sudeste do Brasil,1996 a 1997. Cad Saúde Pública. 2003;19(Suppl 1):S129-S39.

9. Instituto Brasileiro de Geografia e Estatística (IBGE). Pesquisa de Orçamentos Familiares 2008-2009: antropometria e estado nutricional de crianças, adolescentes e adultos no Brasil. Rio de Janeiro: IBGE; 2010.

10. Brasil. Ministério da Saúde. Pesquisa Nacional de Demografia e Saúde da Criança e da Mulher - PNDS 2006: dimensões do processo reprodutivo e da saúde da criança. Centro Brasileiro de Análise e Planejamento. Brasília: Ministério da Saúde, 2009. 300 p.

11. Instituto Brasileiro de Geografia e Estatística (IBGE). Contagem da População 2007. Rio de Janeiro: IBGE; 2007.

12. Gomes RCF, Coelho AAS, Schmitz BAS. Caracterização dos estudos de avaliação do consumo alimentar de pré-escolares. Rev Nutr. 2006;19(6):713-27.

13. Muniz VM. Avaliação dos fatores envolvidos na aceitabilidade do programa de alimentação escolar no município de João Pessoa: um estudo piloto [Dissertação de Mestrado]. João Pessoa (PB): Pós-Graduação em Ciências da Saúde/Universidade Federal da Paraíba; 2006.

14. Segall-Corrêa AM, Marín-León L. A segurança alimentar no Brasil: proposição e usos da Escala Brasileira de Medida da Insegurança Alimentar (EBIA) de 2003 a 2009. Segurança Alimentar e Nutricional. 2009;16(2):1-19. 
15. World Health Organization. Physical Status: the use and interpretation of anthopometry. Geneva; 1995. (WHO Technical Report Series, 854).

16. World Health Organization. AnthroPlus for Personal Computers. Software for assessing growth of the world's children and adolescents. [programa de computador]. Geneva: Department of Nutrition for Health and Development; 2009.

17. Onis M, Onyango AW, Borgui E, Siyam A, Nishida C, Siekmann J. Development of a WHO growth reference for school-aged children and adolescents. Bull World Health Organ. 2007;85(9):660-7.

18. Fonseca VM. Fatores associados ao sobrepeso e obesidade em adolescentes de uma escola privada no município de Niterói [Dissertação de mestrado]. Rio de Janeiro (RJ): Centro Biomédico da Universidade do Estado do Rio de Janeiro/Universidade do Estado do Rio de Janeiro; 1996.

19. Colli AS. Maduración sexual de los adolescentes de São Paulo. In: Organización Panamericana de la Salud. La salud del adolescent y joven en las Americas. Washington, D.C.,1985. p. 249-58. (Publicación científica 489).

20. Duarte MFS. Maturação física: uma revisão na literatura, com especial atenção à criança brasileira. Cad Saúde Pública. 1993;9(Suppl 1):71-84.

21. Centers for Disease Control, World Health Organization. Epi Info, Epidemiologia em microcomputadores: um sistema de processamento de texto, banco de dados e estatística [programa de computador]. Atlanta: OPAS/WHO; 2000.

22. Software de Avaliação Nutricional. Avanutri Online [programa de computador]. Rio de Janeiro: Avanutri Informática Ltda; 2004.

23. Philippi ST. Tabela de composição de alimentos: suporte para decisão nutricional. Coronário: São Paulo; 2002.

24. Universidade Estadual de Campinas. Tabela brasileira de composição de alimentos - TACO. Versão II. 2. ed. 2006.

25. Guimarães LV, Barros MBA. As diferenças de estado nutricional em pré-escolares de rede pública e a transição nutricional. J Pediatr. 2001;77(5):381-6.

26. Anjos LA, Castro IRR, Engstrom EM, Azevedo AMF. Crescimento e estado nutricional em amostra probabilística de escolares no Município do Rio de Janeiro, 1999. Cad Saúde Pública. 2003;19(Suppl 1):171-9.

27. Polla SF, Scherer F. Perfil alimentar e nutricional de escolares da rede municipal de ensino de um município do interior do Rio Grande do Sul. Cad Saúde Colet. 2011;19(1):111-6.

28. Monteiro LN, Aerts D, Zart VB. Estado nutricional de estudantes de escolas públicas e fatores associados em um distrito de saúde do Município de Gravataí, Rio Grande do Sul. Epidemiol Serv Saúde. 2010;19(3):271-81.
29. Oliveira CS, Veiga GV. Estado nutricional e maturação sexual de adolescentes de uma escola pública e de uma escola privada do Município do Rio de Janeiro. Rev Nutr. 2005;18(2):183-91.

30. Linhares RV, Matta MO, Lima JRP, Dantas PMS, Costa MB, Filho JF. Efeitos da maturação sexual na composição corporal, nos dermatóglifos, no somatótipo e nas qualidades físicas básicas de adolescentes. Arq Bras Endocrinol Metab. 2009;53(1):47-54.

31. Waterlow JC. Protein-energy malnutrition: the nature and extent of the problem. Clin Nutr. 1997;16(Suppl 1):3-9.

32. Brasil. Presidência da República. Lei $n^{\circ} 11.947$, de 16 de junho de 2009. Dispõe sobre o atendimento da alimentação escolar e do Programa Dinheiro Direto na Escola aos alunos da educação básica; altera as Leis $\mathrm{n}^{\mathrm{s}} 10.880$, de 9 de junho de 2004, 11.273, de 6 de fevereiro de 2006, 11.507, de 20 de julho de 2007; revoga dispositivos da Medida Provisória no 2.178 -36, de 24 de agosto de 2001, e a Lei ${ }^{\circ}$ 8.913, de 12 de julho de 1994; e dá outras providências. Diário Oficial da União 2009; 17 jun.

33. Andrade RG, Pereira RA, Sichieri R. Consumo alimentar de adolescentes com e sem sobrepeso do Município do Rio de Janeiro. Cad Saúde Pública. 2003;19(5):1485-95.

34. Chaves MGAM, Marques MH, Dalpra JO, Rodrigues PA, Carvalho MF, Carvalho RF. Estudo da relação entre a alimentação escolar e a obesidade. HU Revista. 2008;34(3):191-7.

35. Instituto Brasileiro de Geografia e Estatística (IBGE). Pesquisa de Orçamentos Familiares 2002-2003: Análise da disponibilidade domiciliar de alimentos e do estado nutricional no Brasil. Rio de Janeiro: IBGE; 2004.

36. Instituto Brasileiro de Análises Sociais e Econômicas. Repercussões do Programa Bolsa Família na Segurança Alimentar e Nutricional das Famílias Beneficiadas. Documento Síntese. Rio de Janeiro: Ibase; 2008.

37. Lopes TS. Insegurança alimentar e estado nutricional de adolescentes no distrito Campo Elíseos, município de Duque de Caxias, Rio de Janeiro [Dissertação de mestrado]. Rio de Janeiro (RJ): Universidade Federal do Rio de Janeiro; 2007.

38. Rose D, Oliveira V. Nutrient intakes of individuals from food-insufficient households in the United States. Am J Public Health. 1997;87(12):1956-61.

39. Polivy J. Psychological consequences of food restriction. J Am Diet Assoc. 1996; 96(6):589-92

40. Alaimo K, Olson CM, Frongillo Jr EA. Low family income and food insufficiency in relation to overweight in US children: is there a paradox? Arch Pediatr Adolesc Med. 2001;155(10):1161-7.

Recebido em: 20/04/2012 Aprovado em: 07/04/2013 